\title{
Alta frecuencia del dolor de espalda en los escolares
}

\author{
High frequency of back pain in school children
}

\section{Sr. Editor}

El dolor de espalda es uno de los problemas más comunes de salud en todo el mundo. Se sabe que entre $70 \%$ y $80 \%$ de la población, en algún momento de su vida, experimenta dolor de espalda, cuyas consecuencias se relacionan con limitaciones en las actividades diarias y un alto porcentaje de ausentismo laboral, que genera un impacto económico negativo para el individuo y la sociedad (1).

Diversas investigaciones muestran que los elevados índices de dolor de espalda no solo se presentan en los adultos sino también en los niños y adolescentes (2-6). En un estudio transversal en Polonia en 1224 escolares, se encontró que $74,8 \%$ de estudiantes de entre 10 y 19 años adoleció de dolor de espalda y que este dolor aumentó en relación directa con la edad (4). Asimismo, en otra investigación transversal con 343 escolares de la ciudad de Madrid, se halló que la prevalencia de dolor lumbar fue de $57 \%$ en estudiantes de entre 12 y 15 años, y que la mayor prevalencia se encontraba en el grupo de adolescentes mayores (7). Por otro lado, un estudio de cohorte prospectivo en estudiantes de 11 a 13 años de 14 escuelas de Dinamarca demostró, en un periodo de dos años, no solo que el dolor de espalda es frecuente, sino que el malestar se acrecienta en adolescentes de mayor edad (5).

Cabe mencionar, entonces, que la prevalencia del dolor de espalda en los estudiantes escolares es alta y que progresivamente aumenta con la edad (4-8).
Ahora bien, entre las actividades que desencadenan o aumentan el dolor de espalda en los estudiantes se encuentran la posición sentada por periodos largos durante el horario escolar, postura incorrecta al escribir y usar la computadora, inmobiliario inadecuado, peso y forma de cargar la mochila y la realización de ejercicio físico intenso $(1,6-8)$. Por ello, en el tratamiento del dolor de espalda de los escolares, no solo hay que tener en cuenta el ejercicio fisioterapéutico y la higiene postural (9), sino también evitar los deportes competitivos, el peso excesivo de la mochila y el inmobiliario inadecuado (10). Además, se resalta la importancia de la prevención y tratamiento de esta problemática en etapas tempranas de la vida puesto que los factores asociados al dolor de espalda identificados en la infancia guardan relación con los que se presentan en la edad adulta (2).

Por todo lo mencionado, se concluye que el dolor de espalda puede estar presente en cualquier etapa de la vida, desde la infancia hasta la adultez, siendo necesario tomar medidas tanto preventivas como curativas en etapas tempranas del desarrollo, con el fin de disminuir el avance progresivo de este problema y su influencia en la economía y limitaciones en las actividades diarias de las personas. Además, es importante señalar que se requiere el desarrollo de investigaciones, principalmente, para determinar la prevalencia y los factores desencadenantes del dolor de espalda en los escolares del Perú y la tasa de ausentismo escolar que genera.

Nelly Santos-Cueva ${ }^{1, a}$

\footnotetext{
Departamento de Investigación, Docencia y Rehabilitación Integral en Amputados, Quemados y Trastornos Posturales, Instituto Nacional de Rehabilitación “Doctora Adriana Rebaza Flores” Amistad Perú-Japón. Chorrillos, Perú.

a Tecnólogo Medico en Terapia Física y Rehabilitación
} 


\section{Correspondencia:}

Nelly Santos-Cueva

Correo electrónico: edynelly18@hotmail.com

\section{REFERENCIAS BIBLIOGRÁFICAS}

1. JonesGT,MacfarlaneGJ.Epidemiologyoflowbackpain in children and adolescents. Arch Dis Child. 2005; 90(3):312-6.

2. Muthuri SG, Kuh D, Cooper R. Longitudinal profiles of back pain across adulthood and their relationship with childhood factors: evidence from the 1946 British birth cohort. Pain. 2018; 159(4):764-74.

3. Calvo-Muñoz I, Gómez-Conesa A, Sánchez-Meca J. Prevalence of low back pain in children and adolescents: a meta-analysis. BMC Pediatr. 2013; 13:14.

4. Kędra A, Czaprowski D. Epidemiology of back pain in children and youth aged 10-19 from the area of the southeast of Poland. Biomed Res Int. 2013; 2013: 506823. doi: 10.1155/2013/506823

5. Aartun E, Hartvigsen J, Wedderkopp N, Hestbaek L. Spinal pain in adolescents: prevalence, incidence, and course: a school-based two-year prospective cohort study in 1,300 Danes aged 11-13. BMC Musculoskelet
Disord. 2014; 15:187. doi: 10.1186/1471-2474-15187

6. Noll M, Candotti CT, da Rosa BN, Loss JF. Back pain prevalence and associated factors in children and adolescents: an epidemiological population study. Rev Saúde Pública. 2016; 50:31.

7. Silva MR, Badaró AFV, Dall'Agnol MM. Low back pain in adolescent and associated factors: A cross sectional study with schoolchildren. Braz J Phys Ther. 2014; 18(5): 402-9.

8. Calvo-Muñoz I, Kovacs FM, Roqué M, Gago Fernández I, Seco Calvo J. Risk factors for low back pain in childhood and adolescence: A systematic review. Clin J Pain. 2018; 34(5):468-484. doi: 10.1097/AJP.0000000000000558

9. Calvo-Muñoz I, Gómez-Conesa A, Sánchez-Meca J. Preventive physiotherapy interventions for back care in children and adolescents: a meta-analysis. BMC Musculoskelet Disord. 2012;13:152.

10. Casado M, Moix J, Vidal J. Etiología, cronificación y tratamiento del dolor lumbar. Clínica Salud. 2008; 19(3):379-92.

Recibido: 20/11/2018 